\title{
Alginate/PEG Based Microcarriers with Cleavable Crosslinkage for Expansion and Non-invasive Harvest of Human Umbilical Cord Blood Mesenchymal Stem Cells
}

\author{
Chunge Li ${ }^{\text {a,\#}, \text { Yufeng Qian }}{ }^{\text {b, \#, Shuang Zhao }}$, Yuji Yin ${ }^{\mathrm{a}, *}$, Junjie Li ${ }^{\mathrm{a}, \mathrm{c}, *}$ \\ ${ }^{\mathrm{a}}$ Tianjin Key Laboratory of Composite and Functional Materials, School of Materials Science \\ and Engineering, Tianjin University, No. 92, Weijin Road, Tianjin, 300072, China \\ ${ }^{b}$ Department of Chemistry and Biochemistry, University of Texas at Austin, 2500 Speedway, \\ Austin, TX 78712, USA \\ ${ }^{c}$ Department of Advanced Interdisciplinary Studies, Institute of Basic Medical Sciences and \\ Tissue Engineering Research Center, Academy of Military Medical Sciences, No. 27, Taiping \\ Road, Beijing 100850, China \\ \# These authors contributed equally to this work.
}

*Corresponding authors

Prof. Junjie Li

Tel: +86-10-68166874; Email: li41308@tju.edu.cn; or li41308@aliyun.com Prof. Yuji Yin,

Tel: +86-22-27401902; Fax: +86-22-27404724. Email: yinyuji@tju.edu.cn 


\section{ABSTRACT}

Porous microcarriers are increasingly used to expand and harvest stem cells. Generally, the cells are harvested via proteolytic enzyme treatment, which always leads to damages to stem cells. To address this disadvantage, a series of alginate/PEG (AL/PEG) semi-interpenetrating network microcarriers are prepared in this study. In this AL/PEG system, the chemically cross-linked alginate networks are formed via the reaction between carboxylic acid group of alginate and di-terminated amine groups of cystamine. PEG is introduced to modulate the degradation of microcarriers, which does not participate in this cross-linked reaction, while it interpenetrates in alginate network via physical interactions. In addition, chitosan are coated on the surface of AL/PEG to improve the mechanical strength via the electrostatic interactions. Biocompatible fibronectin are also coated on these microcarriers to modulate the biological behaviors of cells seeded in microcarriers. Results suggest that the size of AL/PEG microcarriers can be modulated via adjusting the contents and molecular weight of PEG. Moreover, the microcarriers are designed to be degraded with cleavage of disulfide crosslinkage. By changing the type and concentration of reductant, the ratio of AL to PEG, and the magnitude of chitosan coating, the degradation ability of AL/PEG microcarriers can be well controlled. In addition, AL/PEG microcarriers can support the attachment and proliferation of human umbilical cord blood mesenchymal stem cells (hUCB-MSCs). More importantly, the expanded hUCB-MSCs can be detached from microcarriers after addition of reductant, which indeed reduce the cell damage caused by proteolytic enzyme treatment. Therefore, it is convinced that AL/PEG based microcarriers will be a promising candidate for large-scale expansion of hUCB-MSCs.

KEYWORDS: Microcarrier, Alginate, PEG, Mesenchymal stem cells, Cell harvesting 


\section{Introduction}

Mesenchymal stem cells (MSCs) have been used as seed cells in cell transplantation and tissue engineering for their good self-renew and differentiation capability. [1] MSCs are typically obtained from bone marrow and adipose. Recently, human umbilical cord blood derived MSCs (hUCB-MSCs) offer a better source due to their non-invasive collection procedure, low risk of infection transmission, hypo-immunogenicity and uperior tropism. [2, 3] In addition, hUCB-MSCs can differentiate into adipocytes, osteoblasts, or chondrocytes as well, [4] which provide a tremendous promise for the treatment of human diseases such as tumor, neurodegenerative disorders, and cardiac diseases. [5, 6] However, the actual number of hUCB-MSCs that can be obtained from donors is still limited compared to the clinical requirements (at a scale of ca. $10^{9}-10^{10}$ cells). [7] Currently, the vast majority of cell expansion methods are mainly based on two-dimension (2D) plastic surface culture in vitro. Such a 2D method with a limited culture area, and consequently a low expansion efficiency, is always labor intensive, time consuming and expensive. Moreover, it is difficult to keep the undifferentiated state for hUCB-MSCs during the frequent subculturing and passage process. Thus, developing an appropriate method for reproducible and scalable harvest of undifferentiated hUCB-MSCs remains a big challenge in the field of cell transplantation and tissue engineering.

Three-dimension (3D) stem cells culture technology with microcarriers was first introduced by van Wezel in 1967, [8] which offered an approach to overcome the restrictions mentioned above. Tan et al., [9] demonstrated that human amniotic MSCs seeded in CultiSpher S microcarriers could quickly proliferate with a high viability. Zhou et al., [10] developed a novel strategy for 3D bone marrow MSCs expansion strategy using Cytodex 3 as the microcarriers. It suggests that the maximum cell density can be achieved at day 5 , which is corresponding to a 
$10.4 \pm 0.8$ fold increases in total cell number. Herein, microcarriers play important roles, which can provide a larger surface area-to-volume ratio (on the order of $4000 \mathrm{~cm}^{2}$ per gram) compared to $2 \mathrm{D}$ planar surface. In addition, some proteins, peptides or growth factors can be incorporated into microcarriers to improve the expansion efficiency. [11] In our previous studies, [12] for example, the fibronectin (Fn) or basic fibroblast growth factor (bFGF) was immobilized on the surface of alginate microcarriers via layer-by-layer technology. Until now, few studies focused on the cell harvesting method from the microcarriers. Proteolytic enzyme (e.g. trypsin, collagenase, and hyaluronidase) treatment is usually employed to detach cells via disrupting cell-matrix interactions from microcarriers. However, the enzymatic digestion process is adverse for the chromosome stability of stem cells and it could decrease the viability and differentiation capacity of stem cells. [13] Moreover, many enzymes employed in these processes are derived from animal sources, which may bring potentially dangerous in the clinical applications. [14] In addition, for the case of stem cells seeded in porous microcarriers, cells are barely accessible to enzymes within a short time, resulting in lots of cells (may be over 40\%) cannot detach from microcarriers, and thus the cell yield obviously decrease. [15] It is necessary to develop novel microcarriers not only have excellent biocompatibility to support the proliferation of stem cells but also can detach the expanded cells via non-enzymatic methods.

To date, the materials of microcarriers are usually comprised of polystyrene, polyethylene, cellulose, gelatin, dextran, chitosan, alginate, etc. In order to harvest cells under mild and non-enzymatic conditions, temperature-responsive poly( $N$-isopropylacrylamide) (PNIPAAm) was coated on these microcarriers surface. [16] Cells can adhere and proliferate on the hydrophobic surface of PNIPAAm based microcarriers when the temperatures is higher than its lower critical solution temperature $\left(\operatorname{LCST}, 32^{\circ} \mathrm{C}\right.$ ) and may be detached spontaneously from the 
hydrophilic surfaces by decreasing the temperature below LCST. [17] For example, Tamura et al., [18] prepared the PNIPAAm-grafted chloromethylated poly(styrene) (CMPS) beads by a surface-initiated atom transfer radical polymerization for facilitating adhesion/detachment of Chinese hamster ovary cells. Previous studies have reported that the detachment efficiency can be promoted by increasing the PNIPAAm content in/on microcarriers, however, the increase of PNIPAAm could also decrease the initial cell adhesion and proliferation efficiency. $[16,19]$ Alternatively, fast degradation of microcarriers is another effective method to harvest the cell. Prestwich et al., [14] prepared hyaluronan and gelatin hydrogel cross-linked by disulfide-containing polyethylene glycol diacrylate for cell expansion. The expanded cell can be collected via the dissociation of the hydrogel by the thiol-disulfide exchange reaction under physiologically conditions. Recently, Jordan et al., [20] also introduced a cleavable disulfide bond into the telechelic poly(2-oxazoline) (POx) crosslinkers and prepared POx-based microbeads by inverse emulsion polymerization. They suggested that the POx-based microbeads can support the proliferation of bone marrow derived human MSCs, moreover, the expanded MSCs can be harvested via the degradation of microbeads by glutathione under mild physiological conditions. In our previous studies, alginate scaffolds [21] and porous microcarriers [12] containing disulfide bonds were prepared via chemically cross-linking by a condensation reaction of cystamine or cysteine with di-terminated amine groups. Results demonstrated that the alginate based microcarriers could support the attachment and proliferation of L929 fibroblasts and human hepatocellular carcinoma cell line HepG2 cells. However, the degradation time of microcarriers was more than $7.5 \mathrm{~h}$, which decreased cell viability and cell yield. Therefore, an alternative microcarrier is demanded for facilitating the cell harvest efficiency as well as maintaining cell adhesiveness. 
In this study, poly (ethylene glycol) (PEG), a well-known water soluble polymer with good biocompatibility and absence of immunogenicity, [22] is incorporated into a chemical cross-linked alginate network containing disulfide bonds to construct the alginate/PEG (AL/PEG) semi-interpenetrating network (semi-IPN) porous microcarriers. To improve the mechanical and biocompatibility properties of microcarriers, chitosan and fibronectin (Fn) were coated on the pore surface of AL/PEG microcarriers via layer-by-layer technology. We focused on the size, morphology, swelling and degradation properties of these microcarriers. Based on these results, seeding efficiency and proliferation behaviors of hUCB-MSCs in AL/PEG microcarriers were evaluated. The cells were harvested by addition of dithiothreitol (DTT), L-cysteine, or glutathione. Furthermore, the stemness and differentiation potential of harvested hUCB-MSCs were also investigated.

\section{Materials and Methods}

\subsection{Materials}

Sodium alginate with a high content of guluronic acid (about $70 \%$ in guluronic acid residues) and a viscosity-average molecular weight of $540 \mathrm{kDa}$ was obtained from FMC Biopolymer (Drammen, Norway). Cystamine di-hydrochloride and PEG with different molecular weight (Mn: 200, 1000, 2050, 4000, 10000) were purchased from Sigma-Aldrich (USA). 1-methyl-3(3-dimethylaminopropyi) carbodiimide hydrochloride $(\mathrm{EDC} \cdot \mathrm{HCl})$ was purchased from GL Biochem (Shanghai, China). Dithiothreitol (DTT), L-cysteine and glutathione were received from Aladdin (Shanghai, China). All other chemicals were of analytical grade and used without further purification.

\subsection{Preparation and surface modification of alginate/PEG microcarriers}


Briefly, $0.60 \mathrm{~g}$ of sodium alginate (AL), a certain amount (cf. Table 1) of PEG with different molecular weights $(\mathrm{Mn})$ and $0.65 \mathrm{~g}$ of $\mathrm{EDC} \cdot \mathrm{HCl}$ were dissolved in $10 \mathrm{~mL}$ of phosphate buffer saline (PBS, $\mathrm{pH}=5.0$ ). The AL/PEG/EDC mixture solutions will be the water phase and Tween 80/peanut oil (1/6, v/v) mixture solution will be the oil phase solution. AL/PEG/EDC mixture solution was added into the oil phase solution under a constant stirring speed (1100 rpm) at $40{ }^{\circ} \mathrm{C}$ for $20 \mathrm{~min}$ (Water phase: Oil phase=1:6 (v/v)). Next, $1 \mathrm{~mL}$ of $\mathrm{EDC} \cdot \mathrm{HCl}$ solution $(1.3$ $\mathrm{g} / \mathrm{mL}$ ) was added into the mixture solution. After $30 \mathrm{~min}, 1.12 \mathrm{~g}$ of cystaminedi-hydrochloride was added into the mixture. The reaction was carried out at $40^{\circ} \mathrm{C}$ under stirring $(1100 \mathrm{rpm})$ for 1 h. AL/PEG semi-IPNs microspheres were obtained after filtration and washed using ethanol.

The prepared microspheres were frozen at $-50{ }^{\circ} \mathrm{C}$ for $24 \mathrm{~h}$ and lyophilized in a freeze dryer to prepare porous AL/PEG semi-IPNs microcarriers. To improve the mechanical strength, chitosan were coated on the surface of porous AL/PEG microcarriers. In brief, AL/PEG-3 microcarriers were immersed in chitosan solution $(3 \mathrm{mg} / \mathrm{ml}$, w/v) at room temperature for $1-3 \mathrm{~h}$ and then soaked in PBS $(\mathrm{pH}=7.4)$ for $48 \mathrm{~h}$ to remove the remaining cross-linking agents and uncoated chitosan. Finally, the chitosan coated AL/PEG (AL/PEG-3/CS) microcarriers were obtained via lyophilization. Fn was further coated on the AL/PEG-3/CS microcarriers to improve the cell attachment capacity. Specifically, AL/PEG-3/CS microcarriers were immersed in Fn solution (4 $\mu \mathrm{g} / \mathrm{mL}$, Biological Industries, Israe) according to our previous study. [12]

\subsection{Characterization of alginate/PEG microcarriers}

The formation of $\mathrm{AL} / \mathrm{PEGs}$ microcarriers was monitored by Fourier Transforms Infrared (FTIR) Spectroscopy (NICOLETNexus-470, USA). The morphologies and size of microspheres were investigated using Culture Microscopes (CHK41, Olympus). Briefly, the AL/PEGs microspheres were uniformly dispersed in water. The size and size distribution of the 
microspheres were analyzed. In addition, the morphology of the AL/PEG and AL/PEG-3/CS microcarriers was characterized using a scanning electron microscope (SEM, PEI Nanosem 430) after sputter-coated by a layer of gold.

\subsection{Swelling and degradation behaviors of AL/PEG microcarriers}

The dried AL/PEG and AL/PEG-3/CS were weighed $(W o$ ) and then were soaked in PBS $(\mathrm{pH}=7.4)$ at $37^{\circ} \mathrm{C}$. After $24 \mathrm{~h}$, the weight $(W t)$ of these microcarriers was accurately measured. The swelling ratio can be calculated using equation (1)

The degradation behavior was investigated by immersing microcarriers into reducing agents solutions with different concentration at $37^{\circ} \mathrm{C}$. The degradation time was defined as the time needed for the samples to be absolutely invisible. Specifically, $0.01 \mathrm{~g}$ of AL/PEG or AL/PEG-3/CS microcarriers were added into $10 \mathrm{~mL}$ of DTT solution $(25 \mathrm{mM})$ to investigate the effects of chemical composition on the degradation behaviors of microcarriers. $0.01 \mathrm{~g}$ of AL/PEG-3 microcarriers were immersed into DTT solution $(10,15,20,25 \mathrm{mM}), L$-cysteine solution $(40,45,50,55 \mathrm{mM})$ or glutathione solution $(80,90100 \mathrm{mM})$, respectively, to investigate the effects of the type and concentration of different reductants on the degradation of microcarriers.

\subsection{Dynamic culture of hUCB-MSCs in Alginate/PEG microcarriers}

Human umbilical cord blood mesenchymal stem cells (hUCB-MSC) were provided by Union Stem Cell \& Gene Engineering Co. Ltd (Tianjin, China). These cells were cultured using DF12 culture media containing $10 \%$ fetal bovine serum and grown at $37^{\circ} \mathrm{C}$ in a humidified atmosphere containing $5 \% \mathrm{CO}_{2}$. The medium was changed twice per week. The $3 \mathrm{rd}$ passage cells were used in the subsequent cultures. 
In order to avoid the hUCB-MSCs adhered on the culture flask, all the culture flasks were siliconized using dimethyldichorosilane solution in carbon tetrachloride (2 wt\%). After washed with deionized water, the siliconized culture flasks were sterilized by autoclaving. The AL/PEG microcarriers were sterilized with $75 \%$ alcohol and then soaked in normal saline. $60 \mathrm{~mL}$ of culture medium containing $0.06 \mathrm{~g}$ AL/PEG microcarriers was added into siliconized culture flask. In order to maximize cell attachment, AL/PEG microcarriers were equilibrated in culture medium for at least 30 min prior to the addition cells. $1.5 \times 10^{6}$ hUCB-MSCs were added into each culture flask and were allowed to attach to in microcarriers undisturbed in a humidified incubator $\left(37^{\circ} \mathrm{C}, 5 \% \mathrm{CO}_{2}\right)$ for $12 \mathrm{~h}$, and then they were cultured on Cell Nest Roller (Shanghai Bio-Tech Co., Ltd). To achieve the suspension culture dynamic environment, the rotation speed is $60 \mathrm{rpm}$.

\subsection{Seeding efficiency and proliferation behaviors of hUCB-MSCs in microcarriers}

At certain time point, $2 \mathrm{~mL}$ of suspension with cell-laden AL/PEG microcarriers was taken out from the culture flask and the number of hUCB-MSC adhered on AL/PEG microcarriers was measured by Cell Counting Kit (CCK-8, DOJINDO Laboratories, Japan) according to the manufacturer's introduction. The seeding efficiency was calculated with equation (2) using the results obtained at day 1 . The proliferation behaviors of hUCB-MSC in microcarriers were evaluated within 7 days. The number of cells at day $0,1,2,4,5,6,7$ was calculated from the standard curve of the absorbance to the number of hUCB-MSCs, and the proliferation behaviors of hUCB-MSCs were measured by the cell number/mg microcarriers.

\subsection{Cell morphology}


CFSE/PI staining was used to investigate the cell viability seeded in microcarriers. Briefly, the cell-laden microcarriers were taken out and transferred to a 24 -well cultured plate at day 1,4 and 7. After washed with PBS ( $\mathrm{pH}=7.4), 500 \mu \mathrm{L}$ of fresh PBS and $4 \mu \mathrm{L}$ of CFSE solution were added into each well and incubated for $20 \mathrm{~min}$. Then, $0.8 \mu \mathrm{L}$ of PI solution was added and incubated for another $10 \mathrm{~min}$. The cell-laden microcarriers were washed twice with PBS $(\mathrm{pH}$ 7.4). Finally, the morphology of hUCB-MSCs in microcarriers was observed by a Nikon 2000 fluorescence microscope. The green living cells were observed at $490 \mathrm{~nm}$ excitation and the red dead cells were seen at $545 \mathrm{~nm}$ excitation. In addition, in order to investigate the distribution of hUCB-MSC in microcarriers, these cell-laden microcarriers were cut into pieces at day 7 and stained using methyl violet staining.

\subsection{Cell harvesting and characterization}

\subsubsection{Cell harvesting}

The expanded hUCB-MSCs were collected when the number of cells reaches its maximum value. Briefly, a certain amount of cell-laden microcarriers were divided and transferred into three centrifuge tubes $(50 \mathrm{~mL})$. DTT, L-cysteine, or glutathione were added to a final concentration of $25 \mathrm{mM}, 55 \mathrm{mM}$ and $100 \mathrm{mM}$, respectively. When all the AL/PEG microcarriers become invisible, the cells were harvested via centrifugation.

\subsubsection{Fluorescence activated cell sorting (FACS) analysis}

The immunophenotype (CD73 PE, CD105 PE, CD34 PE, CD45 PE, HLA-ABC FITC, HLA-DR FITC) of harvested hUCB-MSCs was determined by flow cytometry. Briefly, $100 \mu \mathrm{L}$ of culture medium containing $5 \times 10^{4}$ harvested cells was added into the centrifuge tubes after being washed twice with PBS. $20 \mu \mathrm{L}$ of corresponding antibodies were added into the cell suspension. Mouse IgG1 FITC/Mouse IgG1 was employed as the control antibody. Samples 
were incubated for $30 \mathrm{~min}$ in dark at room temperature. Then, $2 \mathrm{~mL}$ of PBS $(\mathrm{pH}=7.4)$ was added. After centrifuged, the supernatant was discarded and $400 \mu \mathrm{L}$ of paraformaldehyde solutions (1 wt $\%$ ) were added. Finally, the immunophenotype was performed by a flow cytometry (FACS Calibur, BD).

\subsubsection{Self-renewal and differentiation potential of harvested cells}

To investigate the self-renewal and differentiation potential of harvested hUCB-MSCs, they were seeded on 12-well tissue culture polystyrene (TCPS) plate at the seeding density $4 \times 10^{4}$ cells $/ \mathrm{cm}^{2}$, their morphology was observed at day 5 with a light microscopy (Olympus, Japan), and the clone status of hUCB-MSCs was evaluated. The clone numbers of hUCB-MSCs were reported on per $\mathrm{cm}^{2}$. The osteogenic-inducing reagent $(0.05 \mathrm{mM}$ L-ascorbic acid, $10 \mathrm{mM}$ $\beta$-magnesium phosphate pentahydrate and $0.1 \mu \mathrm{M}$ dexamethasone) were added to induce the osteogenic differentiation of harvested hUCB-MSCs. At day 21, the osteogenic differentiation potential was assessed by Von Kossa staining. While, adipogenic differentiation of harvested hUCB-MSCs was induced by adding reagents of $1 \mu \mathrm{M}$ dexamethasone, $0.5 \mathrm{mM}$ isobutylmethylxanthine, and $200 \mu \mathrm{M}$ indomethacin, which was demonstrated by Oil Red O staining at day 14.

\subsection{Statistical analysis}

All data are expressed as mean $\pm \mathrm{SD}$. The data from the five or six groups were compared and inter-group differences were analyzed by one-way ANOVA with Tukey's post-hoc test. Statistical analyses were performed with Origin Pro 8 software. A value of $* p<0.05$ or $* * \mathrm{p}<0.01$ was considered statistically significant.

\section{Results and Discussion}

\subsection{Formation and characterization of alginate/PEG semi-IPNs microcarriers}


First, chemically cross-linked AL/PEG microspheres were prepared via microemulsion technology. Herein, Tween 80/peanut oil was chosen as the oil phase to avoid potential cytotoxicity. In the reaction process, the AL/PEG water solution was added into Tween 80/peanut oil to form the AL/PEG droplets (Fig. 1A). The crosslinker (cystamine) was then added, which can easily go into the AL/PEG droplets due to its excellent water solubility. The crosslink reaction occurs between the carboxylic acid group of alginate and di-terminated amine groups of cystamine, resulting in the formation of cross-linked alginate network linked by the amide linkage (Fig. 1A). As shown in Fig. 1B, the absorption bands of amide groups at 1620 $\mathrm{cm}^{-1}$ appear in all samples, indicating that the formation of chemically cross-linked alginate network. Here, PEG does not participate in this cross-linked reaction, while it interpenetrates in alginate network via physical interactions.

The molecular weight $(\mathrm{Mn})$ and content of PEG can influence the morphology of AL/PEG microspheres. As shown in Fig. 2D, the microspheres are uniform spherical when the $M n$ of PEG is 2050, while microspheres become ellipsoidal when the $M n$ of PEG is 200, 1000, 4000 and 10000 (Fig. 2B, 2C, 2E \& 2F). It is well known that the water solubility of PEG decreases with the increase of molecular weight of PEG. For the PEG with large molecular weight ( $\geq 4000$ ), the PEG may leak from AL/PEG droplets and come into the oil phase solution in the preparation process, which may result in the AL/PEG microsphere become irregular. For the PEG with small molecular weight $(\leq 1000)$, the PEG can release form AL/PEG microspheres when they are washed using water, which can destroy the morphology of the microsphere. In comparison with microspheres formed with AL only (Fig. 2A), similar microspheres can be formed with AL/PEG ratio of 1:1, which are spherical, colorless, transparent, high moisture content, soft, and elastic 
(Fig. 2D). With increasing the content of PEG to a ratio of 1:1.5, however, microspheres become irregular (Fig. 2G). For the AL/PEG ratio of 1:2, only some gel fragments can be found (Fig. $2 \mathrm{H})$. We conclude that the increase of PEG content is adverse for the formation of AL/PEG microspheres. In addition, the adding of PEG can increase the size of microspheres. The size of length direction and width direction of most $\mathrm{AL}$ microspheres ranges 900-1800 $\mu \mathrm{m}$ and 500-1100 $\mu \mathrm{m}$, respectively (Fig. 2I and J). The size of AL/PEG-3 microspheres is 1100-1900 $\mu \mathrm{m}$ and 700-1200 $\mu \mathrm{m}$ (Fig. 2M and N). It is believed that a high content of PEG may decrease the crosslinking degree and the tightness of microspheres, resulting in the size increase of the AL/PEG droplets.

The porous AL/PEG microcarriers are formed after freeze-drying and their morphologies are shown in Fig. 3. The lyophilization time affects morphology of microcarriers. Compared to microcarriers that lyophilized only once (Fig. 3A-C), the morphology of microcarriers experienced twice lyophilization become messy (Fig. 3D \& 3F). This phenomenon may be caused by following reason, when the dry microcarriers experienced once lyophilization are immersed in water, they can absorb lots of water and swell. During the process of swelling, the molecular chain of PEG and alginate rearrange and become more regularity. These behaviors result in the microcarriers become messy after lyophilization twice times. The content of PEG is also an important parameter to modulate the morphology of microcarriers, as shown in Fig. 3A-3C and 3G-3I, the surface morphology of microcarriers become well-structured when the ratio of AL/PEG varies from 1:0 to 1:1. After being lyophilized twice, this phenomenon is more prominent (Fig. 3D-3F and 3J-3L). In addition, the surface modification using chitosan makes microcarriers become more regular (Fig. 3J-3L). We reason that the chitosan coating could improve the mechanical strength due to the electrostatic interactions between amine groups of 
chitosan and carboxylic acid groups of alginate. It should be noted that the pore size of all microcarriers ranges 20-200 $\mu \mathrm{m}$, which is suitable for the growth of hUCB-MSCs in three-dimensionally dynamic culture. [23]

\subsection{Swelling properties of microcarriers}

Although PEG molecular chains are not involved in the cross-linking reaction of the AL network in the microcarriers, they affect the swelling ratio of AL/PEG microcarriers. [24] As shown in Fig. 4, all AL/PEG microcarriers exhibit high water-absorbing capacity, which is beneficial for cell adhesion and growth, and transport of nutrients from the microcarriers to cells in vitro. [25] Additionally, our data suggest that the chemical composites and surface modification are the main factors to influence the swelling ratio of microcarriers. Fig. 4A displays the swelling ratios of $\mathrm{AL} / \mathrm{PEG}$ microcarriers with different $\mathrm{AL} / \mathrm{PEG}$ ratios. The swelling ratio of AL microcarriers is $6.7 \pm 0.06$, which increases to $8.21 \pm 0.12$ and $8.38 \pm 0.01$ when the AL/PEG ratio is $1: 0.5$ and 1:1 $(p<0.01)$, respectively. Tanuma et al., [26] also found that the swelling ratio of the chitosan/PEG composites increases with the increasing of PEG content. It is reasonable to invoke the hydrophilic nature of PEG. A hydration layer can be formed on PEG chain through hydrogen bonds. [27] The incorporation of PEG makes the chemically cross-linked AL network become loose and a reduction of intramolecular hydrogen bonds among AL molecules, which results in the entrance of more water molecules into the microcarriers. However, the swelling ratios begin to decrease when $\mathrm{AL} / \mathrm{PEG}$ ratio goes beyond 1:1. In AL/PEG microcarriers with high PEG content, the chemically cross-linked AL network can not bound all PEG molecules. Therefore, some PEG may dissolve and release out of microcarriers due to the high water-solubility, leading to a decline in swelling ratio. As to surface modification, our results show that the chitosan coating can decrease the water adsorption capacity of AL/PEG 
microcarriers and the swelling ratio lightly decrease with the increase of modification time (Fig. 4B). It is interpreted that the chitosan modification can enhance the mechanical strength of the microcarriers, which strengthens the ability of microcarriers to maintain their original shapes. [12]

\subsection{Degradation behaviors of microcarriers}

The stability of AL/PEG microcarriers was evaluated in water at $37^{\circ} \mathrm{C}$. Results suggest that the AL/PEG microcarriers with low PEG content, namely with AL/PEG ratio more than 1:1, are stable for more than 15 days. Nonetheless, they disappear and become homogeneous clear solution when a suitable amount of reductant was added. During this process, the covalently cross-linked AL networks are de-crosslinked by a reductant (such as, DTT, L-cysteine, glutathione), which can cleave the -S-S- bond in the covalent linkages between alginate chains (Fig. 5A). The mechanism of this reaction is similar to that of the thiolated alginate reported by Bernkop-Schnürch et al. [28]

The type and concentration of reductant are the main factors to modulate the degradation of AL/PEG microcarriers. Fig. 5B-5D sums the degradation times of AL/PEG-3 microcarriers in DTT, L-cysteine, and glutathione solution with different concentrations. Results indicate the degradation time decreases with the increasing of the concentration of the reductant. All the AL/PEG-3 microcarriers degrade within $65 \mathrm{~min}$ in $10 \mathrm{mM}$ DTT solution and the degradation time decrease to $27.75 \pm 1.70 \mathrm{~min}$ when the concentration of DTT increases to $25 \mathrm{mM}$ (Fig. 5B). The degradation time of microcarriers is $90.00 \pm 1.41$ and $81.25 \pm 1.70 \mathrm{~min}$ in $45 \mathrm{mM}$ and 50 mM L-cysteine solution, respectively. However, when the concentration of L-cysteine is less than $40 \mathrm{mM}$, the degradation seems to be suspended (Fig. 5C). For the glutathione, a large number of microcarriers always cannot be degraded within $240 \mathrm{~min}$ even to the glutathione is 
endowed a concentration of $100 \mathrm{mM}$ (Fig. 5D). The degradation behaviors also depend on the content of PEG in microcarriers, as shown in Fig. 5E. All the AL microcarriers degrade after 66 \pm 1.29 min when immersed in DTT solution with a concentration of $25 \mathrm{mM}$. The addition of PEG can accelerate the degradation of microcarriers, for example, the degradation time of $\mathrm{AL} / \mathrm{PEG}-2$ and AL/PEG-3 is $(55 \pm 0.81)$ and $(27.75 \pm 1.70)$ min, respectively, which is significantly lower than that of $\mathrm{AL}(\mathrm{p}<0.05$ and $\mathrm{p}<0.01)$. The decreasing of crosslinking density in microcarriers with the increase of PEG content is the main reason. However, the AL/PEG microcarriers exhibit similar degradation behaviors with $\mathrm{AL}$ microcarriers when the $\mathrm{AL} / \mathrm{PEG}$ ratio is less than 1:1. This trend is consistent with that of swelling behaviors. The higher the swelling ratio is, the more reductant can enter into the semi-IPN AL/PEG network microcarriers, which indeed enhances the degradation reaction.

In addition, the modification of chitosan also influences the degradation behaviors of AL/PEG microcarriers as well. As shown in Fig. 5F, the degradation time of microcarriers in DTT solution $(25 \mathrm{mM})$ gradually increases from $27.75 \pm 1.70 \mathrm{~min}$ to $122 \pm 0.81 \mathrm{~min}$ with the increase of the modification time $(0-3 \mathrm{~h})$. Notably, the degradation time after modified for $3 \mathrm{~h}$ is significantly higher than that others $(\mathrm{p}<0.01)$. Chitosan coating reduces the contact probability of reductant and disulfide bonds, resulting in the decrease of de-crosslinking. The surface coating also prevents the release of water-soluble PEG molecules from microcarriers, as mentioned above in the discussion of swelling behaviors. The magnitude of chitosan coating increases with the modification time. And thus, the degradation rate of AL/PEG microcarriers can be well controlled via this method.

\subsection{Expansion of hUCB-MSCs in alginate/PEG microcarriers}


According to our previous study, [12] chitosan and Fn were successively immobilized on the surface of porous AL/PEG microcarriers via layer-by-layer (LBL) self-assembly technology before seeding hUCB-MSCs. We proposed that the chitosan coating can improve the mechanical rigidity and bio-adhesive properties of microcarriers, and the Fn layer is beneficial to the expansion of hUCB-MSCs. As shown in Fig. 6A, we found that the seeding efficiency in AL and AL/PEG-3 is $(68.23 \pm 0.12) \%$ and $(68.16 \pm 0.32) \%$, respectively, indicating that the addition of PEG does not affect the seeding efficiency in microcarriers. After coated with chitosan and chitosan/Fn, the seeding efficiency increases to $(76.24 \pm 0.43) \%$ and $(79.81 \pm 0.28) \%$, respectively, which is significantly higher than that of in AL and AL/PEG-3 microcarriers $(\mathrm{p}<0.01)$. The less seeding efficiency of AL and AL/PEG-3 microcarriers is mainly caused by the absence of cell-surface receptors on alginate and/or PEG chains. [29] While, after modified with chitosan or chitosan/Fn, lots of positive charges $\left(-\mathrm{NH}_{3}{ }^{+}\right)$appear on the surface of microcarriers, which facilitates the attachment of negatively charged cells. [30] Moreover, Fn is able to specifically interact with cells through its arginine-glycine-aspartic (RGD) sequence, [31] which effectively improves the seeding efficiency of hUCB-MSCs in microcarriers.

To determine the cell expansion properties of AL/PEG microcarriers, hUCB-MSCs seeded in microcarriers were cultured within 7 days under dynamic condition. As shown in Fig. 7, few dead cells (stained with red color) can be observed in all microcarriers, indicating that the AL/PEG microcarriers prepared in this study have no cytotoxicity. Moreover, the fluorescence intensities of the cells (stained green) are not identical, which is due to the cells are existed in different layer of microcarriers. In order to further investigate the distribution of cells in microcarriers, cell-laden microcarriers were cut into pieces and stained using methyl violet staining, as shown Fig. S1, the cell can be observed in the inner of microcarrier, especially for 
AL/PEG-3/CS and AL/PEG-3/CS/Fn microcarriers, indicating that the hUCB-MSCs grown not only on the microcarriers' surface but also in the interiors of microcarriers. In addition, the number of hUCB-MSCs on all microcarriers rapidly increases to $1.1-1.3 \times 10^{4}$ from ca. $1 \times 10^{3}$, and there are no significantly differences among these groups (Fig. 6B and Fig. 7). However, the number of cells in $\mathrm{AL}$ and $\mathrm{AL} / \mathrm{PEG}$ microcarriers began to decrease after 2 days, because these microcarriers have no enough mechanical strength to support the cell adhesion. Especially for the AL/PEG microcarriers, its mechanical strength decreases when the non-crosslinked PEG molecules release from the microcarriers during the culture process. Our previous study [12] showed that the microcarriers after modified with chitosan have enough mechanical strength to support the adhesion of cells, but this study finds the number of cells in AL/PEG-CS microcarriers keep almost unchanged after 2 days. It is because the high interactions between negative cell and positive chitosan layer. [32] As expected, the cells in AL/PEG-CS/Fn microcarriers continue to proliferate after 2 days and the proliferation rate of cells on them is obviously higher than that in other microcarriers $(\mathrm{p}<0.01)$. Furthermore, the cell number arrives at the maximum $\left(1.66 \times 10^{4}\right)$ after 5 days of culture, which is equivalent to more than 12 -fold of cell expansion. These cells may have occupied all accessible surfaces of the microcarriers and thus resulting in the death of cells at day 6 and 7. These results are similar with Yang's report [33]. They found that after 4 days' suspension culture in spinner flasks, the embryonic stem cells could be expanded to 12.5 fold in number, however, extending the culturing time to 6 days caused significant loss in cell viability. Zhou et al., [10] also found that the maximum density of bone marrow MSCs on Cytodex 3 microcarriers can be achieved at day 5, corresponding to a $10.4 \pm 0.8$ fold increases in total cell number. In addition, the doubling time of hUCB-MSCs is 55.56 and $34.58 \mathrm{~h}$ in AL/PEG-3/CS and AL/PEG-3/CS/Fn microcarriers, respectively, which is 
consistent with the previous study. [34] The biocompatible Fn coating is the main reason to reduce the doubling time. Above results indicating that AL/PEG-3CS/Fn microcarriers would be an attractive candidate for the cell expansion of stem cell, although their cell expanding capacity should be further improved.

\subsection{Harvesting and characterization of hUCB-MSCs}

Non-invasive cell harvesting is an advantageous method for collecting large-scale intact cells. Fig. 8 shows the mechanism of non-invasive cell harvest from AL/PEG microcarriers. The AL/PEG semi-IPN network would be de-crosslinked when the -S-S- bonds are cleaved by reductant. Then due to the good water solubility, AL and PEG could dissolve in water and simultaneously the cells can be detached from the microcarriers. In order to explain the advantages of non-invasive cell harvest method, the traditional enzyme digestion was also used to detach the cell form AL/PEG-3/CS/Fn microcarriers as control. Results suggest that the traditional enzyme treatment results in a low harvesting efficiency of less than $10.6 \%$, which is similar with previous study reported. [35] One possible reason for this low efficiency is that most cells are grown in the inner of microcarriers, and thus many of the cells may not contact with the enzyme and the detached cells may not remove from the microcarriers. As expected, the harvesting efficiency obviously increases after the degradation of microcarriers. The microcarriers completely degraded after the addition of reductant (DTT and L-cysteine), most cells can be detached from the microcarriers and harvested. Approximate $85 \%$ of cells are harvested within 30 min after addition of DTT, and 70\% cells can detach from all microcarriers within 60 min after addition of L-cysteine. This detachment efficiency is similar with other non-enzymatic harvest methods. For example, Tamura et al., [16] showed that the detachment efficiency of Chinese hamster ovary cells form thermally responsive microcarriers depended on 
the brush density of PNIPAAm on microcarriers surface. It was more than $77 \%$ for microcarriers with dense PNIPAAm brushed and the microcarriers with intermediate brush density exhibited the highest detachment efficiency. Herein, few cells can be harvested after the addition of glutathione due to the low degradation efficiency.

Furthermore, the self-renewal of harvested cells is investigated. Cells were seeded on the TCPS and their statuses were observed using microscope at day 5. Fig. S2 shows the monolayer culture of the hUCB-MSCs after being collected using DTT or L-cysteine, which demonstrates the retention of the cells' ability to attach and proliferate on TCPS. Although the high cell harvesting efficiency is obtained within 30 min using DTT due to its excellent reductive capacity, the cells are injured to some extent owing to the cytotoxicity of DTT. As shown in Table 2, the number of cell clones, which are collected from AL/PEG-3 microcarriers using DTT as the reactant, is only ca. 5 . The chitosan and chitosan/Fn modification can reduce the cell damage, clones of cells detached from AL/PEG-3/CS and AL/PEG-3/CS/Fn is ca. 14 and 16, respectively. Both L-cysteine and glutathione have excellent biocompatibility. Therefore, the cells detached from all microcarriers using L-cysteine exhibit excellent viability (clones > 20) although its harvesting efficiency is lower than that using DTT. However, the clone statuses are not observed after harvesting using glutathione due to its low reductive capacity. From this study, L-cysteine can be deemed as a most suitable reducing agent to harvest the expanded cells form AL/PEG microcarriers via non-enzymatic digestion method.

The stemness of harvested cells is an important index for a stem cell expansion technology. Previous studies have suggested that a non-enzymatic harvesting procedure would be preferable approach to preserve the immunophenotype of the cell. $[35,36]$ The immunophenotype of harvested hUCB-MSC from AL/PEG-3/CS/Fn microcarriers using L-cysteine was determined by 
flow cytometry. As shown in Fig. 9A, the harvested cells show strong positivity to CD73, CD105, and HLA-ABC, but are negative to CD34, CD45, and HLA-DR. These data are consistent with immunophenotypic markers of MSCs, [37, 38] implying that the harvesting procedure via degradation of microcarriers does not change the immunophenotype of hUCB-MSCs. In addition, in order to investigate the differentiation potential of hUCB-MSC after harvesting, they were cultured on TCPS under adipogenic or osteogenic microenvironment. As shown in Fig. 9B, the harvested cells gradually become shorter spindle from long fusiform shape under adipogenic differentiation conditions and become elliptical and circular at day 14 . Moreover, the lipid droplets and positive Oil Red "O" staining can be observed. On the other hand, light brown to dark brown precipitates are formed in cytoplasm under osteogenic induced conditions (Fig. 9C). These results prove that the harvested hUCB-MSCs maintain the stemness and have adipogenic and osteogenic differentiation potential. [39, 40]

\section{Conclusions}

A series of novel porous alginate/PEG semi-IPN microcarriers with cleavable crosslinkage were successfully developed for expansion and non-invasive harvest of human umbilical cord blood mesenchymal stem cells. Current study is being conducted to investigate the modulation conditions and degradation behaviors of microcarriers. Results suggest that the morphology and size of AL/PEG microcarriers strongly depended on the content and molecular weight of PEG in microcarriers and the modification on the surface of microcarriers. Furthermore, the AL/PEG microcarriers can be degraded via cleaving the -S-S- bonds using reductant. AL/PEG microcarriers can support the attachment and expansion of hUCB-MSCs. More importantly, the expanded cells can be harvested via degradation of microcarriers, which could reduce the cell damage caused by traditional proteolytic enzyme treatment. The cells harvested from 
AL/PEG-3/CS/Fn microcarriers by L-cysteine not only have excellent viability but also maintain the stemness and differentiation potential. We anticipate that our developed AL/PEG based microcarriers will be of practical value in the large-scale stem cell culture for tissue engineering and cell transplantation.

\section{Declaration of interest}

The authors state no conflict of interest in this study.

\section{Acknowledgments}

This project was supported by the National Science Foundation of China via Grants 31370975, 51073116, and 31100674; National High Technology Research and Development Program of China (2015AA020304); Beijing Natural Science Foundation (No. 7162150).

\section{References}

[1]. M. D. Hoffman, A. H. Van Hove, D. S. W. Benoit, Degradable hydrogels for spatiotemporal control of mesenchymal stem cells localized at decellularized bone allografts, Acta Biomater. 10 (2014) 3431-3441.

[2]. M. M .Lalu, L. McIntyre, C. Pugliese, D. Fergusson, B. W. Winston, J. C. Marshall, J. Granton, D. J. Stewart, Canadian critical care trials, g. safety of cell therapy with mesenchymal stromal cells (safecell): a systematic review and meta-analysis of clinical trials, PLoS One 7 (2012) e47559.

[3]. M. Lee, S. Y. Jeong, J. Ha, M. Kim, H. J. Jin, S. J. Kwon, J. W. Chang, S. J. Choi, W. Oh, Y. S. Yang, J. S. Kim, H. B. Jeon, Low immunogenicity of allogeneic human umbilical cord blood-derived mesenchymal stem cells in vitro and in vivo, Biochem. Biophys. Res. Commun. 446 (2014) 983-989. 
[4]. M. Zarrabi, S. H. Mousavi, S. Abroun, B. Sadeghi, Potential uses for cord blood mesenchymal stem cells, Cell 15 (2014) 274-181.

[5]. E. J. Lee, S. J. Park, S. K. Kang, G. H. Kim, H. J. Kang, S. W. Lee, H. B. Leo, H. S Kim, Spherical bullet formation via E-cadherin promotes therapeutic potency of mesenchymal stem cells derived from human umbilical cord blood for myocardial infarction, Mol. Ther. 20 (2012) 1424-1433.

[6]. J. C. Ra, I. S. Shin, S. H. Kim, S. K. Kang, B. C. Kang, H. Y. Lee, Y. J. Kim, J. Y. Jo, E. J. Yoon, H. J. Choi, E. Kwon, Safety of intravenous infusion of human adipose tissue-derived mesenchymal stem cells in animals and humans, Stem Cells Dev. 20 (2011) 1297-1308.

[7]. A. K. L. Chen, S. Reuveny, S. K. W. Oh, Application of human mesenchymal and pluripotent stem cell microcarrier cultures in cellular therapy: achievements and future direction, Biotechnol. Adv. 31 (2013) 1032-1046.

[8]. A. L. van Wezel, Growth of cell-strains and primary cells on micro-carriers in homogeneous culture, Nature 216 (1967) 64-65.

[9]. M. Q. Chen, X. Wang, Z. Y. Ye, Y. Zhang, Y. Zhou, W. S. Tan, A modular approach to the engineering of a centimeter-sized bone tissue construct with human amniotic mesenchymal stem cells-laden microcarriers, Biomaterials 32 (2011) 7532-7542.

[10]. L. Zhou, J. Kong, Y. Zhuang, J. Chu, S. Zhang, M. Guo, Ex vivo expansion of bone marrow mesenchymal stem cells using microcarrier beads in a stirred bioreactor, biotechnol, Bioprocess Eng. 18 (2013) 173-184. 
[11]. J. H. Lee, J. H. Park, A. El-Fiqi, J. H. Kim, Y. R. Yun, J. H. Jang, C. M. Han, E. J. Lee, H. W. Kim, Biointerface control of electrospun fiber scaffolds for bone regeneration: engineered protein link to mineralized surface, Acta Biomater. 10 (2014) 2750-2761.

[12]. C. Li, S. Zhao, Y. Zhao, Y. Qian, J. Li, Y. Yin, Chemically crosslinked alginate porous microcarriers modified with bioactive molecule for expansion of human hepatocellular carcinoma cells, J. Biomed. Mater .Res. Part B Appl. Biomater.102 (2014) 1648-1658.

[13]. M. M. Mitalipova, R. R. Rao, D. M. Hoyer, J. A. Johnson, L. F. Meisner, K. L. Jones, S. Dalton, S. L. Stice, Preserving the genetic integrity of human embryonic stem cells, Nat. Biotechnol. 23 (2005) 19-20.

[14]. J. Zhang, A. Skardal, G. D. Prestwich, Engineered extracellular matrices with cleavable crosslinkers for cell expansion and easy cell recovery, Biomaterials 29 (2008) 4521-4531.

[15]. S. Bancel, W. S. Hu, Confocal laser scanning microscopy examination of cell distribution in macroporous microcarriers, Biotechnol. Progress. 12 (1996) 398-402.

[16]. A. Tamura, J. Kobayashi, M. Yamato, T. Okano, Temperature-responsive poly (N-isopropylacrylamide)-grafted microcarriers for large-scale non-invasive harvest of anchorage-dependent cells, Biomaterials 33 (2012) 3803-3812.

[17]. J. N. Zhang, Z. F. Cui, R. Field, M. G. Moloney, S. Rimmer, H. Ye, Thermo-responsive microcarriers based on poly(N-isopropylacrylamide). Eur Polym J 67 (2015) 346-364.

[18]. A. Tamura, J. Kobayashi, M. Yamato, T. Okano, Thermally responsive microcarriers with optimal poly(N-isopropylacrylamide) grafted density for facilitating cell adhesion/detachment in suspension culture. Acta Biomater 8 (2012) 3904-3913.

[19]. A. Tamura, M. Nishi, J. Kobayashi, K. Nagase, H. Yajima, M. Yamato, T. Okano, Simultaneous Enhancement of Cell Proliferation and Thermally Induced Harvest 
Efficiency Based on Temperature-Responsive Cationic Copolymer-Grafted Microcarriers. Biomacromolecules 13 (2012) 1765-1773.

[20]. S. Lück, R. Schubel, J. Rüb, D. Hahn, E. Mathieu, H. Zimmermann, D. Scharnweber, C. Werner, S. Pautot, R. Jordan, Tailored and biodegradable poly(2-oxazoline) microbeads as 3D matrices for stem cell culture in regenerative therapies, Biomaterials, 79 (2016) 1-14.

[21]. Y. Zhao, S. Gao, S. Zhao, Y. Li, L. Cheng, J. Li, Y. Yin, Synthesis and characterization of disulfide-crosslinked alginate hydrogel scaffolds, Mater. Sci. Eng. C 32 (2012) 2153-2162.

[22]. C. Wu, A. Ying, S. Ren, Fabrication of polymeric micelles with core-shell-corona structure for applications in controlled drug release, Colloid Polym. Sci. 291 (2013) 827-834.

[23]. F. G. Thankam, J. Muthu, Alginate based hybrid copolymer hydrogels-influence of pore morphology on cell-material interaction, Carbohyd. Polym.112 (2014) 235-244.

[24]. A. Jejurikar, X. T. Seow, G. Lawrie, D. Martin, A. Jayakrishnan, L. Grondahl, Degradable alginate hydrogels crosslinked by the macromolecular crosslinker alginate dialdehyde, J. Mater. Chem. 22 (2012) 9751-9758.

[25]. P. Jithendra, A. M. Rajam, T. Kalaivani, A. B. Mandal, C. Rose, Preparation and characterization of aloe vera blended collagen-chitosan composite scaffold for tissue engineering applications, ACS Appl. Mater. Interfaces 5 (2013) 7291-7298.

[26]. H. Tanuma, T. Saito, K. Nishikawa, T. Dong, K. Yazawa, Y. Inoue, Preparation and characterization of peg-cross-linked chitosan hydrogel films with controllable swelling and enzymatic degradation behavior, Carbohyd. Polym.80 (2010) 260-265.

[27]. K. Susumu, E. Oh, J. B. Delehanty, F. Pinaud, K. B. Gemmill, S. Walper, J. Breger, M. J. Schroeder, M. H. Stewart, V. Jain, C. M. Whitaker, A. L. Huston, I. L. Medintz, A new 
family of pyridine-appended multidentate polymers as hydrophilic surface ligands for preparing stable biocompatible quantum dots, Chem. Mater.26 (2014) 5327-5344.

[28]. A. Bernkop-Schnurch, C. E. Kast, M. F. Richter, Improvement in the mucoadhesive properties of alginate by the covalent attachment of cysteine, J. Controlled Release 71 (2001) 277-285.

[29]. R. Tan, Z. She, M. Wang, Z. Fang, Y. Liu, Q. Feng, Thermo-sensitive alginate-based injectable hydrogel for tissue engineering, Carbohyd. Polym.87 (2012) 1515-1521.

[30]. J. J. Li, Y. Shu, T. Hao, Y. Wang, Y. Qian, C. Duan, H. Sun, Q. Lin, C. Wang, A chitosan-glutathione based injectable hydrogel for suppression of oxidative stress damage in cardiomyocytes, Biomaterials 34 (2013) 9071-9081.

[31]. A. Dolatshahi-Pirouz, T. Jensen, D. C. Kraft, M. Foss, P. Kingshott, J. L. Hansen, A. N. Larsen, J. Chevallier, F. Besenbacher, Fibronectin adsorption, cell adhesion, and proliferation on nanostructured tantalum surfaces, ACS Nano 4 (2010) 2874-2882.

[32]. M. Prasitsilp, R. Jenwithisuk, K. Kongsuwan, N. Damrongchai, P. Watts, Cellular responses to chitosan in vitro: the importance of deacetylation, J. Mater. Sci. Mater. Med. $11(2000) 773-778$.

[33]. N. Liu, Y. Li, S. T. Yang, Expansion of embryonic stem cells in suspension and fibrous bed bioreactors, J. Biotechnol. 178 (2014) 54-64.

[34]. E. Y. L. Fok, P. W. Zandstra, Shear-controlled single-step mouse embryonic stemcell expansion and embryoidbody-based differentiation, Stem Cell 23 (2005)1333-1342.

[35]. A. W. Nienow, Q. A. Rafiq, K. Coopman, C. J. Hewitt, A potentially scalable method for the harvesting of hmscs from microcarriers, Biochem. Eng. J. 85 (2014) 79-88. 
[36]. S. Jung, K. M. Panchalingam, R. D. Wuerth, L. Rosenberg, L. A. Behie, Large-scale production of human mesenchymal stem cells for clinical applications, Biotechnol. Appl. Biochem. 59 (2012) 106-120.

[37]. X. Zhang, M. Hirai, S. Cantero, R. Ciubotariu, L. Dobrila, A. Hirsh, K. Igura, H. Satoh, I. Yokomi, T. Nishimura, S. Yamaguchi, K. Yoshimura, P. Rubinstein, T. A. Takahashi, Isolation and characterization of mesenchymal stem cells from human umbilical cord blood: reevaluation of critical factors for successful isolation and high ability to proliferate and differentiate to chondrocytes as compared to mesenchymal stem cells from bone marrow and adipose tissue, J. Cellular Biochem.112 (2011) 1206-1218.

[38]. Y. Han, J. Chai, T. Sun, D. Li, R. Tao, Differentiation of human umbilical cord mesenchymal stem cells into dermal fibroblasts in vitro, Biochem. Biophys. Res. Commun. 413 (2011) 561-565.

[39]. S. Roy, S. Arora, P. Kumari, M. Ta, A simple and serum-free protocol for cryopreservation of human umbilical cord as source of wharton's jelly mesenchymal stem cells, Cryobiology 68 (2014) 467-472.

[40]. P. Phuc Van, V. Ngoc Bich, P. Vuong Minh, T. NhungHai, P. Truc Le-Buu, D. Loan Thi-Tung, N. Tam Thanh, B. Anh Nguyen-Tu, P. Ngoc Kim, Good manufacturing practice-compliant isolation and culture of human umbilical cord blood-derived mesenchymal stem cells, J. Translational Med.12 (2014) 56. 


\section{Figure Captions}

Fig. 1 (A) Schematic representation of formation process of AL/PEG hydrogel microspheres, (B) FTIR spectra of AL and AL/PEG microcarriers with different molecular weight of PEG.

Fig. 2 Morphology of AL/PEG microspheres, (A) AL, (B) AL/PEG-6 (PEG 200), (C) AL/PEG-7 (PEG 1000), (D) AL/PEG-3 (PEG 2050), (E) AL/PEG-8 (PEG 4000) and (F) AL/PEG-9 (PEG 10000), (G) AL/PEG-5 (1:1.5) and (H) AL/PEG-5 (1:2); Size distribution of AL (I, J), AL/PEG-2 (K, L) and AL/PEG-3 (M, N) in length direction and width direction, respectively.

Fig. 3 SEM images of AL/PEG microcarriers. (A) AL, (B) AL/PEG-2 and (C) AL/PEG-3 microcarriers after once lyophilization; (D) AL, (E) AL/PEG-2 and (F) AL/PEG-3 microcarriers after twice lyophilization; (G) AL, (H) AL/PEG-2 and (I) AL/PEG-3 microcarriers modified with chitosan after once lyophilization; (J) AL, (K) AL/PEG-2 and (L) AL/PEG-3 microcarriers modified with chitosan after twice lyophilization.

Fig. 4 (A) Swelling ratios of AL/PEG microcarriers with different AL/PEG ratio; (B) Swelling ratio of AL microcarriers after modification in chitosan solution for $1-3 \mathrm{~h}$. $(* \mathrm{p}<0.05, * * \mathrm{p}<0.01)$

Fig. 5 (A) Schematic representation of the degradation mechanisms of AL/PEG microcarriers; Degradation time of AL/PEG-3 microcarriers in (B) DTT, (C) L-cysteine and (D) glutathione solution with different concentration; (E) Effect of AL/PEG ratio on degradation behaviors of microcarriers; (F) Degradation time of AL/PEG-3/CS microcarriers. ( ${ }^{*} p<0.05$, ${ }^{*} \mathrm{p}<0.01$, NS: no statistical significance)

Fig. 6 (A) Seeding efficiency of hUCB-MSCs in microcarriers at day 1; (B) Proliferation curves of hUCB-MSCs seeded in microcarriers within 7 days. $\left({ }^{*} p<0.05, * * p<0.01\right.$, NS: no statistical 
differences, $\bullet \mathrm{p}<0.05, \mathrm{AL} / \mathrm{PEG}-\mathrm{CS} / \mathrm{Fn}$ group vs. AL/PEG group, $\# \mathrm{p}<0.01, \mathrm{AL} / \mathrm{PEG}-\mathrm{CS} / \mathrm{Fn}$ group vs. other group)

Fig. 7 CFSE/PI staining of hUCB-MSCs seeded in microcarriers at day 1, 4 and 7. Bar=100 $\mu \mathrm{m}$

Fig. 8 Schematic representation of detachment mechanisms of hUCB-MSCs induced by a degradation of AL/PEG microcarriers.

Fig. 9 (A) Flow cytometric analysis of hUCB-MSCs harvested from AL/PEG-3/CS/Fn using L-cysteine. The harvested hUCB-MSCs are positive for CD73, CD105 and HLA-ABC for characteristic of mesenchymal stem cells, but negative for CD34, CD45, and HLA-DR; (B) Oil Red O staining of harvested hUCB-MSC for adipogenic differentiation; (C) Von Kossa staining of harvested hUCB-MSC for osteogenic differentiation.

\section{Table Captions}

Table 1. Preparation Parameter of Alginate/PEG semi-IPNs Microspheres

Table 2. The number of clones of harvested cells from AL/PEG microcarriers 


\section{Figures}


Fig. 1 (A) Schematic representation of formation process of AL/PEG hydrogel microspheres, (B) FTIR spectra of AL and AL/PEG microcarriers with different molecular weight of PEG. 

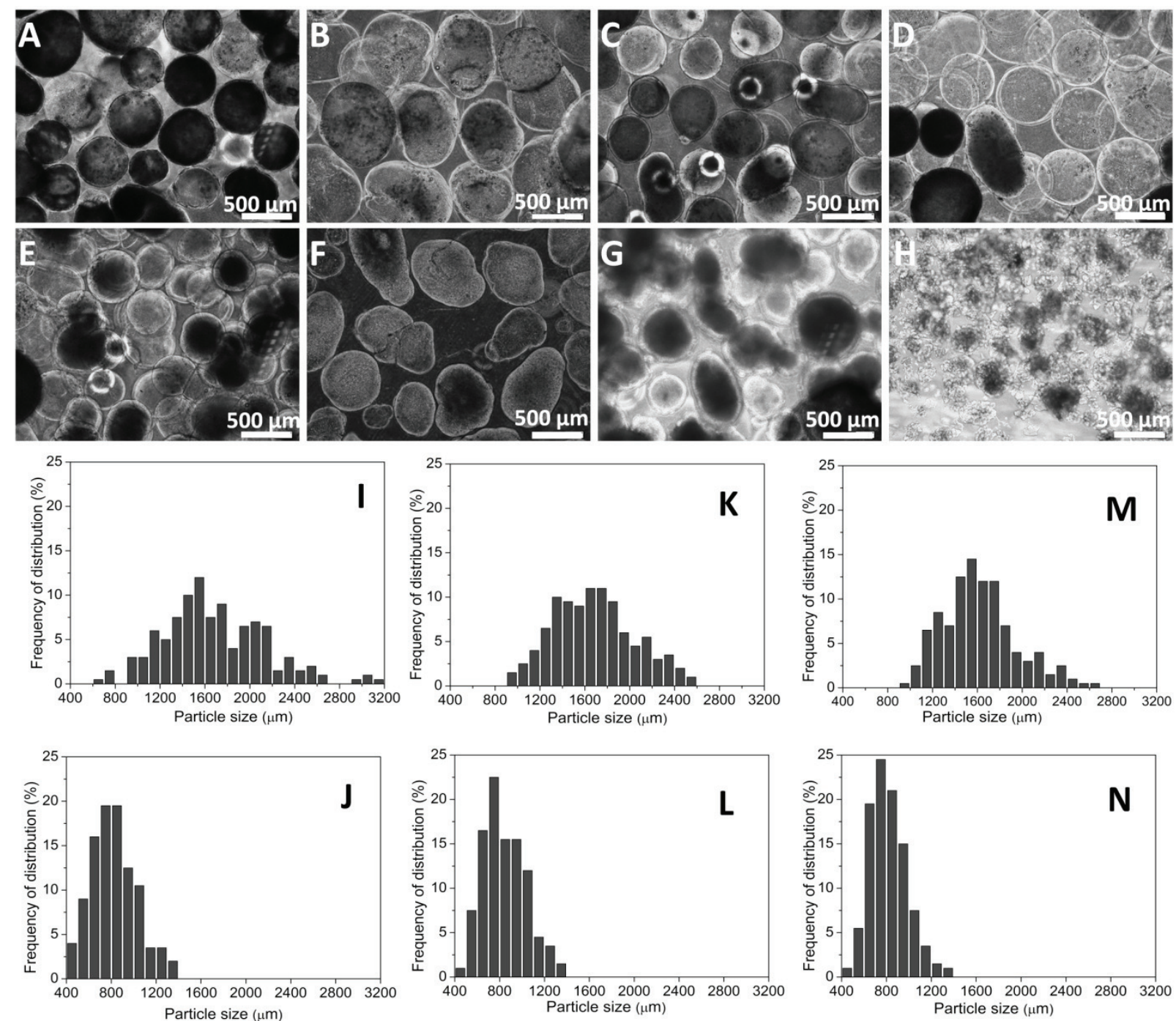

Fig. 2 Morphology of AL/PEG microspheres, (A) AL, (B) AL/PEG-6 (PEG 200), (C) AL/PEG-7 (PEG 1000), (D) AL/PEG-3 (PEG 2050), (E) AL/PEG-8 (PEG 4000) and (F) AL/PEG-9 (PEG 10000), (G) AL/PEG-5 (1:1.5) and (H) AL/PEG-5 (1:2); Size distribution of AL (I, J), AL/PEG-2 (K, L) and AL/PEG-3 (M, N) in length direction and width direction, respectively. 



Fig. 3 SEM images of AL/PEG microcarriers. (A) AL, (B) AL/PEG-2 and (C) AL/PEG-3 microcarriers after once lyophilization; (D) AL, (E) AL/PEG-2 and (F) AL/PEG-3 microcarriers after twice lyophilization; (G) AL, (H) AL/PEG-2 and (I) AL/PEG-3 microcarriers modified with chitosan after once lyophilization; (J) AL, (K) AL/PEG-2 and (L) AL/PEG-3 microcarriers modified with chitosan after twice lyophilization. 

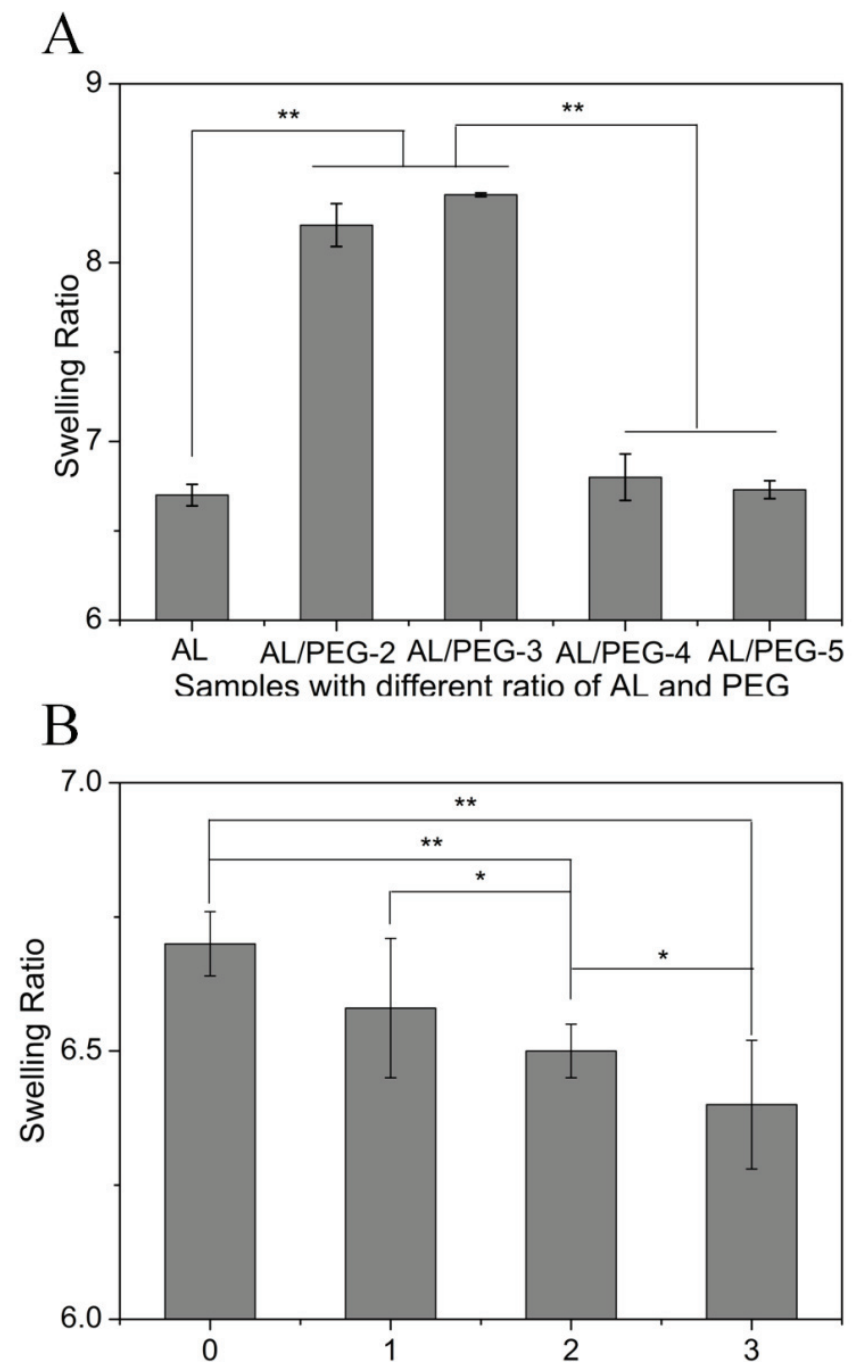

The time of AL microcarriers soaked in chitosan solution (h)

Fig. 4 (A) Swelling ratios of AL/PEG microcarriers with different AL/PEG ratio; (B) Swelling ratio of AL microcarriers after modification in chitosan solution for $1-3 \mathrm{~h} .(* \mathrm{p}<0.05, * * \mathrm{p}<0.01)$ 


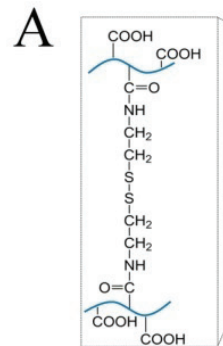

B

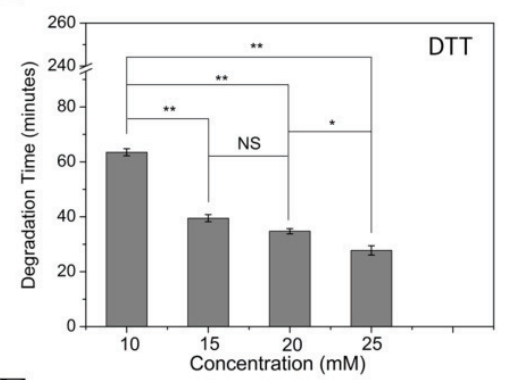

E

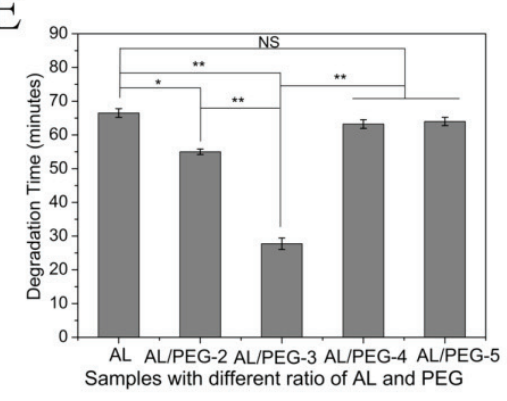

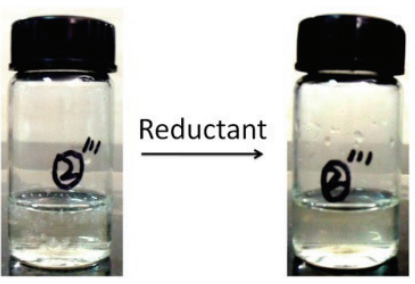

AL and PEG solution



$\mathrm{C}$

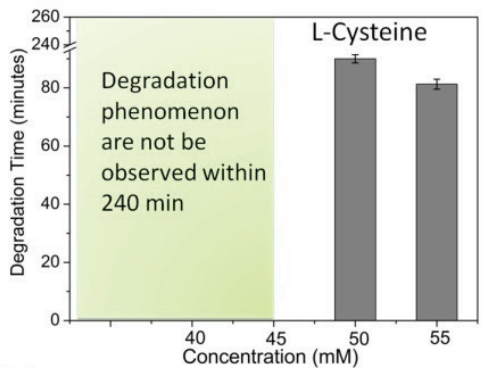

D
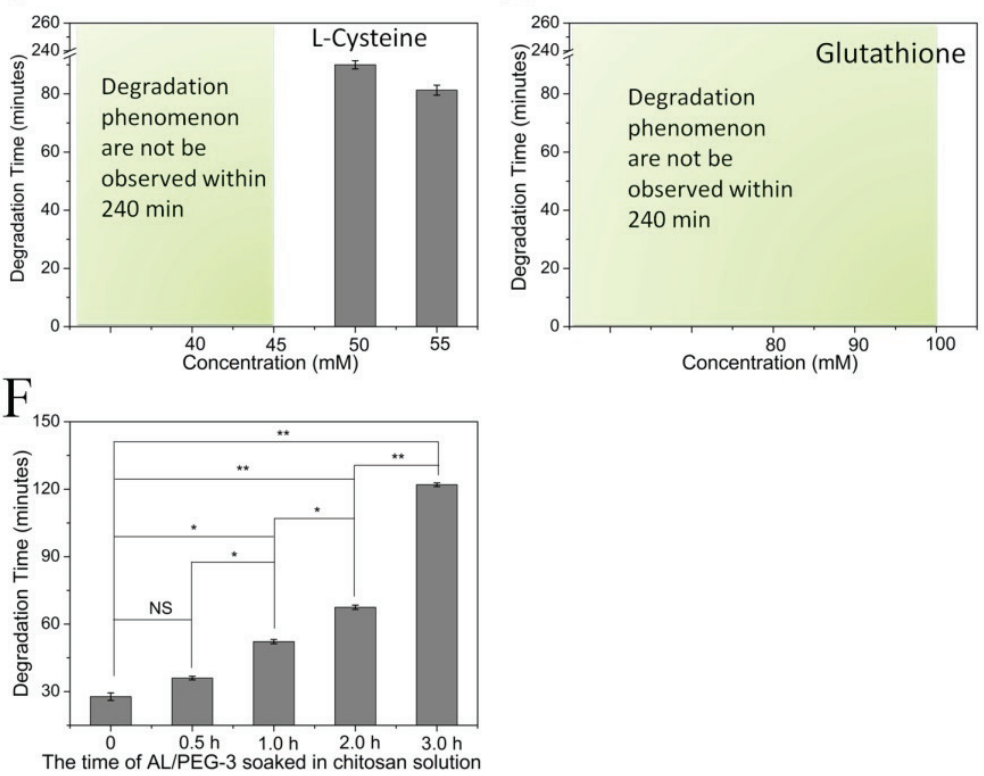

Fig. 5 (A) Schematic representation of the degradation mechanisms of AL/PEG microcarriers; Degradation time of AL/PEG-3 microcarriers in (B) DTT, (C) L-cysteine and (D) glutathione solution with different concentration; (E) Effect of AL/PEG ratio on degradation behaviors of microcarriers; (F) Degradation time of AL/PEG-3/CS microcarriers. $\left({ }^{*} \mathrm{p}<0.05,{ }^{*} \mathrm{p}<0.01\right.$, NS: no statistical significance) 

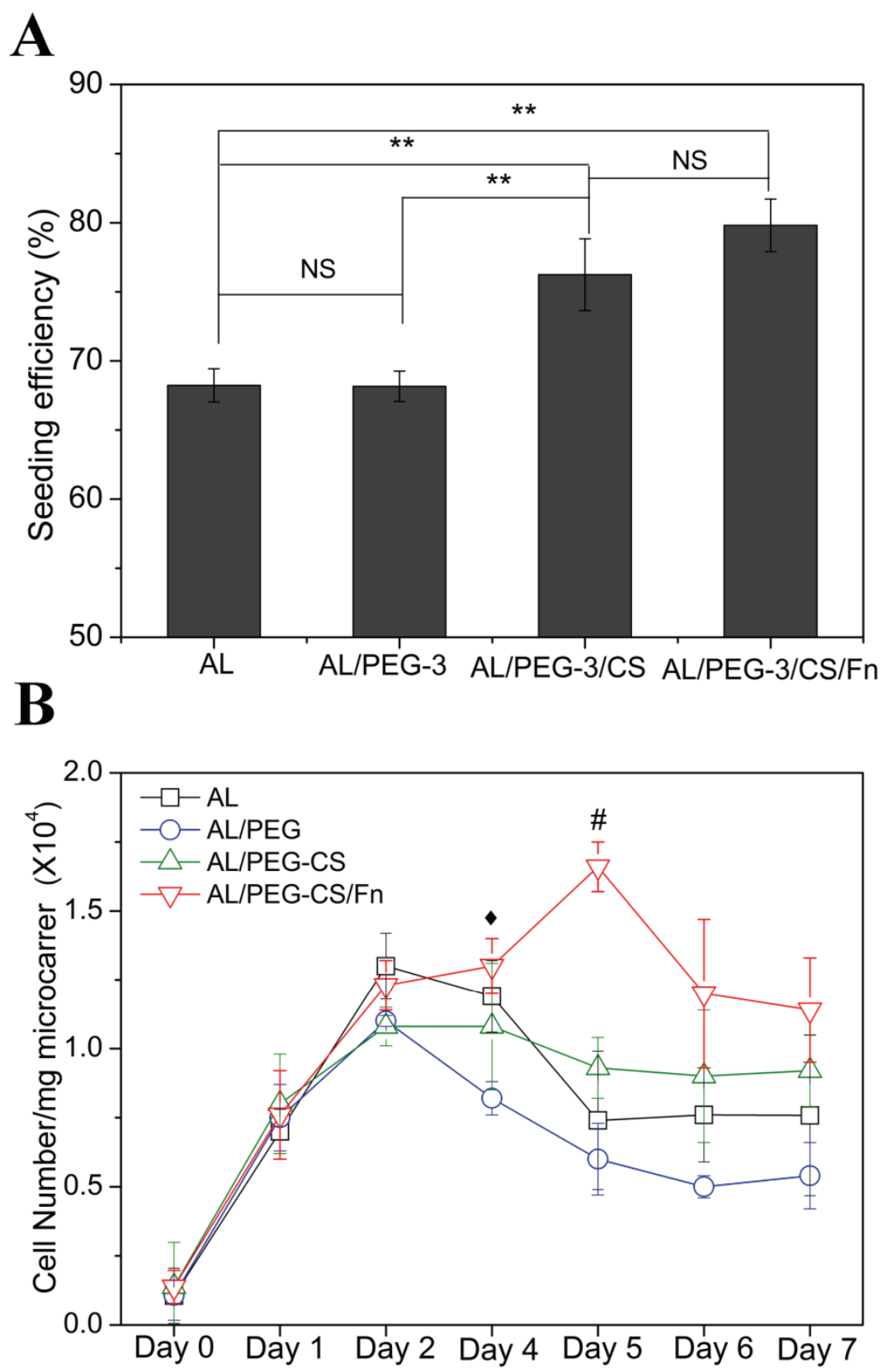

Fig. 6 (A) Seeding efficiency of hUCB-MSCs in microcarriers at day 1; (B) Proliferation curves of hUCB-MSCs seeded in microcarriers within 7 days. $\left({ }^{*} p<0.05, * * p<0.01\right.$, NS: no statistical differences, $\downarrow \mathrm{p}<0.05, \mathrm{AL} / \mathrm{PEG}-\mathrm{CS} / \mathrm{Fn}$ group vs. AL/PEG group, $\# \mathrm{p}<0.01, \mathrm{AL} / \mathrm{PEG}-\mathrm{CS} / \mathrm{Fn}$ group vs. other group) 


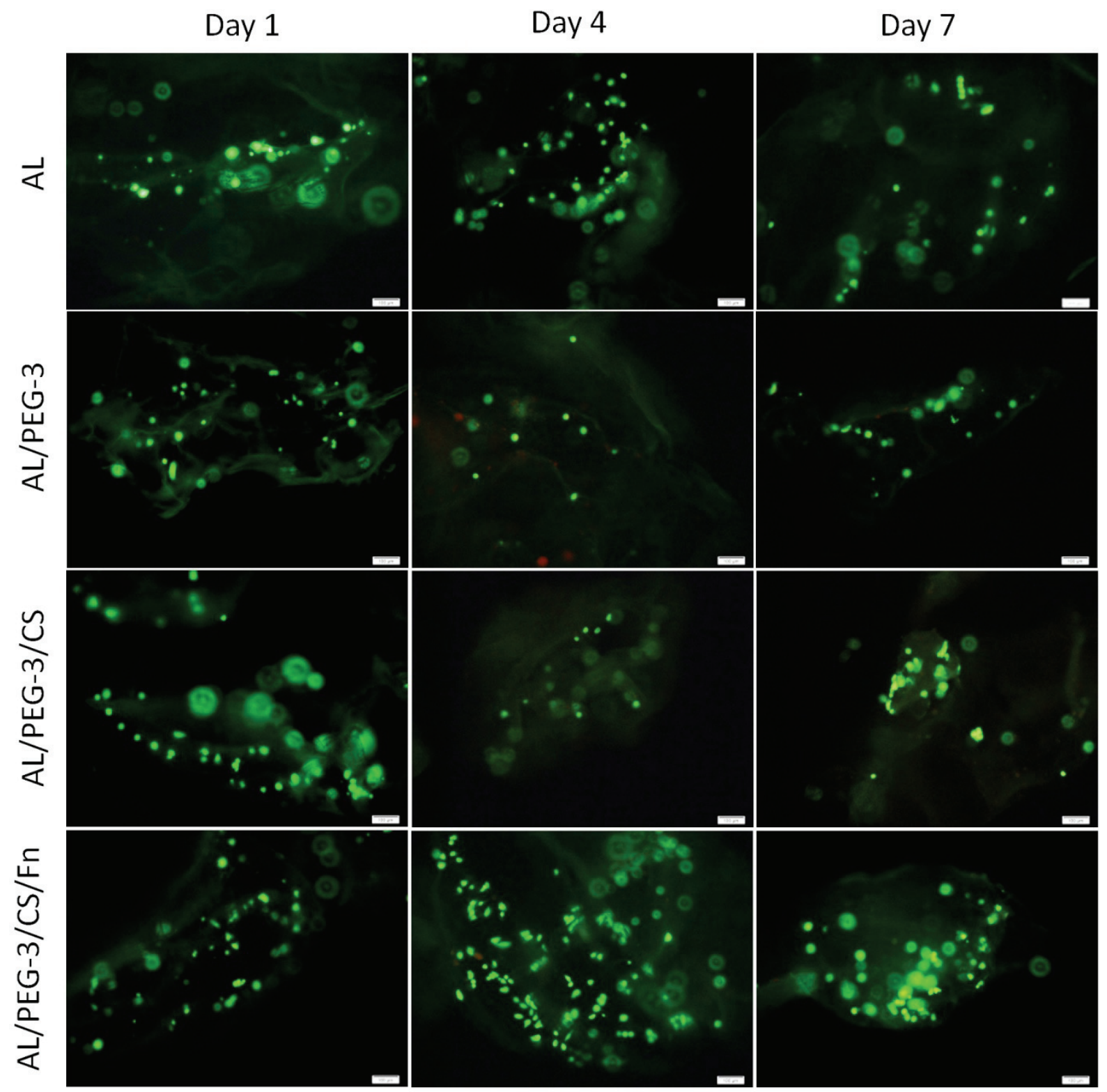

Fig. 7 CFSE/PI staining of hUCB-MSCs seeded in microcarriers at day 1, 4 and 7. Bar=100 $\mu \mathrm{m}$ 




Cell adhesion and expansion on porous AL/PEG microcarriers

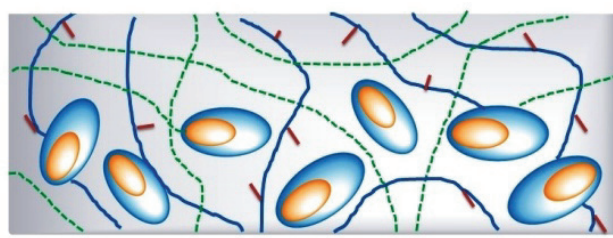

AL/PEG microcarriers degraded and cell harvesting

Fig. 8 Schematic representation of detachment mechanisms of hUCB-MSCs induced by a degradation of AL/PEG microcarriers. 

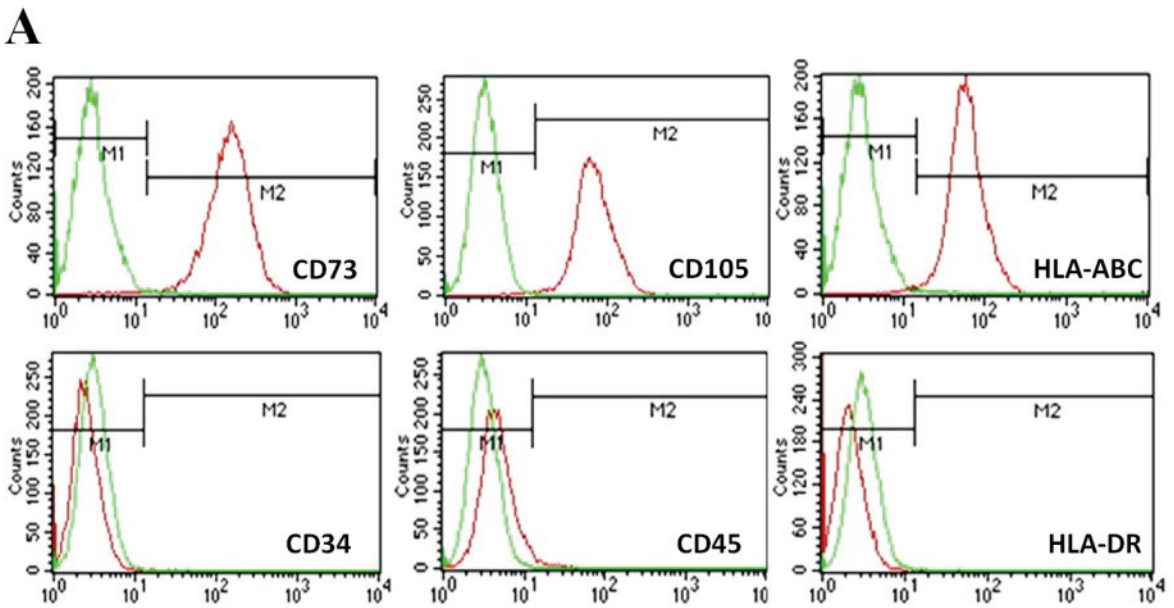

B
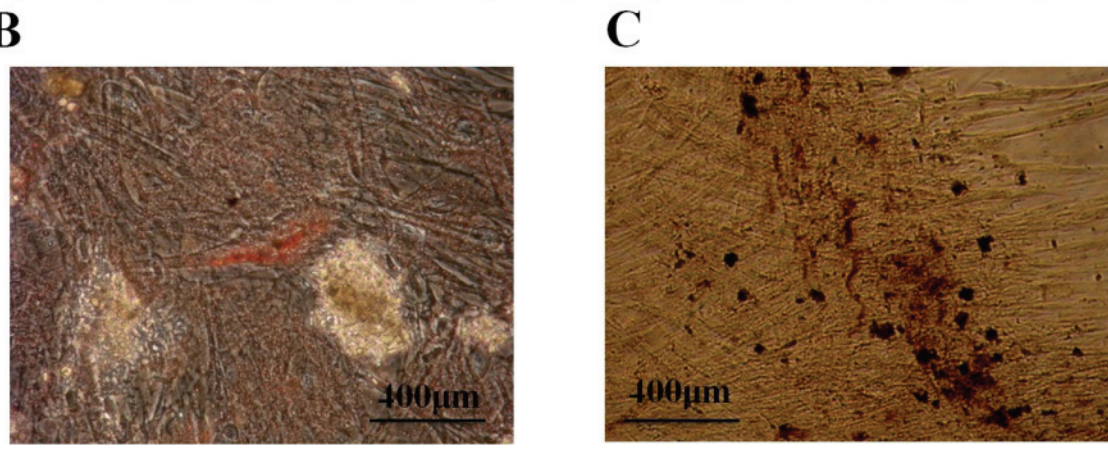

Fig. 9 (A) Flow cytometric analysis of hUCB-MSCs harvested from AL/PEG-3/CS/Fn using L-cysteine. The harvested hUCB-MSCs are positive for CD73, CD105 and HLA-ABC for characteristic of mesenchymal stem cells, but negative for CD34, CD45, and HLA-DR; (B) Oil Red O staining of harvested hUCB-MSC for adipogenic differentiation; (C) Von Kossa staining of harvested hUCB-MSC for osteogenic differentiation. 


\section{Tables}

Table 1. Preparation Parameter of Alginate/PEG semi-IPNs Microspheres

\begin{tabular}{lll}
\hline Sample & Mn of PEG & Alginate/PEG (g/g) \\
\hline AL & & \\
AL/PEG-2 & 2050 & $1: 0$ \\
AL/PEG-3 & 2050 & $1: 0.5$ \\
AL/PEG-4 & 2050 & $1: 1$ \\
AL/PEG-5 & 2050 & $1: 1.5$ \\
AL/PEG-6 & 200 & $1: 2$ \\
AL/PEG-7 & 1000 & $1: 1$ \\
AL/PEG-8 & 4000 & $1: 1$ \\
AL/PEG-9 & 10000 & $1: 1$ \\
\hline
\end{tabular}

Table 2. The number of clones of harvested cells from AL/PEG microcarriers

\begin{tabular}{llll}
\hline \multirow{2}{*}{ Microcarriers } & \multicolumn{2}{l}{ Number of clones $/ \mathrm{cm}^{2}$} & \\
\cline { 2 - 4 } & DTT & L-Cysteine & Glutathione \\
\hline AL & 4 & $>20$ & N. D. \\
AL/PEG-2 & 4 & $>20$ & N. D. \\
AL/PEG-3 & 5 & $>20$ & N. D. \\
AL/PEG-3/CS & 14 & $>20$ & N. D. \\
AL/PEG-3/CS/Fn & 16 & $>20$ & N. D. \\
\hline
\end{tabular}

